\title{
FDTD BASED SECOND-ORDER ACCURATE LOCAL MESH REFINEMENT METHOD FOR MAXWELL'S EQUATIONS IN TWO SPACE DIMENSIONS *
}

\author{
A. R. ZAKHARIAN ${ }^{\dagger}$, M. BRIO $\ddagger$, AND J. V. MOLONEY $\S$
}

\begin{abstract}
An algorithm is presented for local space-time mesh refinement appropriate for electromagnetic simulations based on the space-time staggered FDTD method. The method is based on the adaptive mesh refinement algorithm originally developed for hyperbolic conservation laws. Analysis of the dispersion relation and of the numerical reflection and transmission coefficients in one and two space dimensions shows that a scheme based on linear interpolation at the grid interfaces is unstable due to reflection coefficient $>1$ at frequencies above the cutoff frequency of the coarse grid. A second-order accurate algorithm based on higher-order interpolations that enforces conservation of the magnetic field circulation at the fine-coarse grid boundaries is constructed. The new algorithm is shown to be stable and accurate for long time integration. A numerical simulation of an optical ring microcavity resonator using multilevel grid refinement in two space dimensions is presented.
\end{abstract}

Key words. computational electrodynamics, Maxwell's equations, subgridding, AMR, grid interfaces, FDTD, mesh refinement, numerical simulation, optical waveguide

AMS subject classifications. 65M06, 65M50, 78M20

\section{Introduction}

In the last two decades there have been numerous investigations of the local grid refinement strategies. The main advantages of such methods are improved resolution and efficiency over the methods that use uniform mesh size. The trade off is in a more complicated data structure and a need for interface boundary conditions. The adaptive mesh refinement algorithm (AMR) developed originally by Berger et al (e.g. [1] and references therein) for systems of hyperbolic conservation laws is freely available in a software package AMRCLAW [1]. The algorithm found many applications in fluid dynamics, magnetohydrodynamics and astrophysics [6].

In this article we extend the AMR algorithm to the space-time staggered Yee FDTD scheme for Maxwell's equations of electrodynamics. The key issue is the development of the fine-coarse boundary condition that is void of spurious oscillations, accurate and stable for long time integration with small but fixed mesh sizes, and not just in the theoretical limit of vanishing discretization parameters. For example, if a reflection coefficient at the interface is $R=1+O(\Delta t)$, the theoretical convergence is not precluded according to Lax convergence theory, as $\Delta t \rightarrow 0$. In practice, however, such $R>1$ may lead to instability if $\Delta t$ stays small but fixed and the number of reflections is sufficiently large.

Numerical studies of this issue performed in the past [5], [8] indicated that the stability of the local grid refinement algorithm is sensitive to the interpolation method and the choice of the interpolated fields. In [3] the problem of spurious reflections at the grid interfaces with material traverse was addressed by a set of experimentally

\footnotetext{
* Received: May 11, 2004; accepted (in revised version): August 17, 2004. Communicated by Shi Jin.

†University of Arizona, Optical Sciences Center and ACMS, Dept. of Math., Tucson, AZ 85721, USA (armis@email.arizona.edu).

$\ddagger$ University of Arizona, ACMS, Dept. of Math., Tucson, AZ 85721, USA (brio@math.arizona.edu).

$\S$ University of Arizona, Optical Sciences Center and ACMS, Dept. of Math., Tucson, AZ 85721, USA (jml@acms.arizona.edu).
} 
determined averaging procedures, illustrating that indeed the filtering of the high frequencies may work.

The theoretical analysis of a reflection coefficient for the scheme that preserves discrete energy was presented in [4] for a one-dimensional case. The reflection and transmission coefficients were shown to be of order one near Nyquist frequencies and the overall scheme is consistent and convergent in the theoretical limit of vanishing discretization parameters. However, the discrete energy defined in [4] is invariant for arbitrary CFL numbers, and does not preclude amplification of the high frequency modes.

For semi-discrete approximation with continuous time and discrete space [7] and [10] showed that it is possible to construct an interpolation scheme at the grid interfaces, such that the energy is conserved in continuous time.

Finally, a paper by Vay [11] compares various reflection coefficients in the context of a new numerical scheme proposed by the author. The method consists of replacing the wave equation by a set of characteristic equations describing left and right going waves and then applies the one-sided Sommerfeld boundary condition for each of the waves. The author showed a significant reduction in the reflection coefficient in comparison to previously suggested methods.

In this article we propose formulation of the AMR technique for the space-time staggered Yee FDTD algorithm that enforces the conservation of circulation in a manner similar to the conservation of the flux in hyperbolic conservation laws. The method fits into the standard AMR grid management scheme and allows for the use of previously developed software. Numerical examples illustrating accuracy, stability, and efficiency are performed on three problems: scattering from a dielectric cylinder, propagation in a waveguide and computation of coupling efficiency in a microring cavity.

In the following section we describe the dispersion relation for the Yee scheme and the evanescent, non-propagating modes with complex wave numbers. Interface boundary conditions are derived and studied analytically and numerically in sections 2.2 and 2.3. Numerical examples are presented in section 3.

\section{FDTD grid refinement algorithm}

2.1. The Yee scheme dispersion relation. We consider the nondimensional linear vector Maxwell's equations in isotropic, homogeneous nondispersive media for transverse magnetic (TM) mode propagation (ignorable z-coordinate),

$$
\begin{aligned}
& \frac{\partial H_{x}}{\partial t}=-\frac{\partial E_{z}}{\partial y}, \quad \frac{\partial H_{y}}{\partial t}=\frac{\partial E_{z}}{\partial x}, \\
& \frac{\partial E_{z}}{\partial t}=\frac{\partial H_{y}}{\partial x}-\frac{\partial H_{x}}{\partial y} .
\end{aligned}
$$

Numerical discretization on each level of refinement is done using the standard Yee scheme that is staggered in space and time and applied on a uniform mesh,

$$
\begin{array}{r}
H_{x i+1 / 2, j}^{n+1 / 2}-H_{x i+1 / 2, j}^{n-1 / 2}=-\nu_{y}\left(E_{z i+1 / 2, j+1 / 2}^{n}-E_{z i+1 / 2, j-1 / 2}^{n}\right), \\
H_{y i, j+1 / 2}^{n+1 / 2}-H_{y i, j+1 / 2}^{n-1 / 2}=\nu_{x}\left(E_{z i+1 / 2, j+1 / 2}^{n}-E_{z i-1 / 2, j+1 / 2}^{n}\right), \\
E_{z i+1 / 2, j+1 / 2}^{n+1}-E_{z i+1 / 2, j+1 / 2}^{n}=\nu_{x}\left(H_{y i+1, j+1 / 2}^{n+1 / 2}-H_{y i, j+1 / 2}^{n+1 / 2}\right) \\
-\nu_{y}\left(H_{x i+1 / 2, j+1}^{n+1 / 2}-H_{x i+1 / 2, j}^{n+1 / 2}\right),
\end{array}
$$


where $\nu_{x}$ and $\nu_{y}$ are defined as $\Delta t / \Delta x$ and $\Delta t / \Delta y$, respectively. A diagram of the staggered grid of the Yee scheme is shown in Fig. 2.1 along with variable locations at the interface between coarse and fine grids. The grid is usually refined near the material interfaces and in the regions of high refractive index, where the wavelength is shorter, and hence higher resolution is beneficial. We restrict our analysis to the cases when material interfaces do not cross grid refinement interfaces. The reflection and transmission properties of such grid interfaces will be modified, and this analysis is out of the scope of the present paper. However, in the numerical examples we applied our algorithm also to the cases of grid interface crossing the material boundary.

When the material interfaces are enclosed by the refined grids, the appropriate FDTD update for Maxwell's equations with inhomogeneous material properties can be applied at the material interfaces. Therefore, at the grid refinement interfaces, we can use in the following analysis the equations (2.1)-(2.3) valid for homogeneous medium.

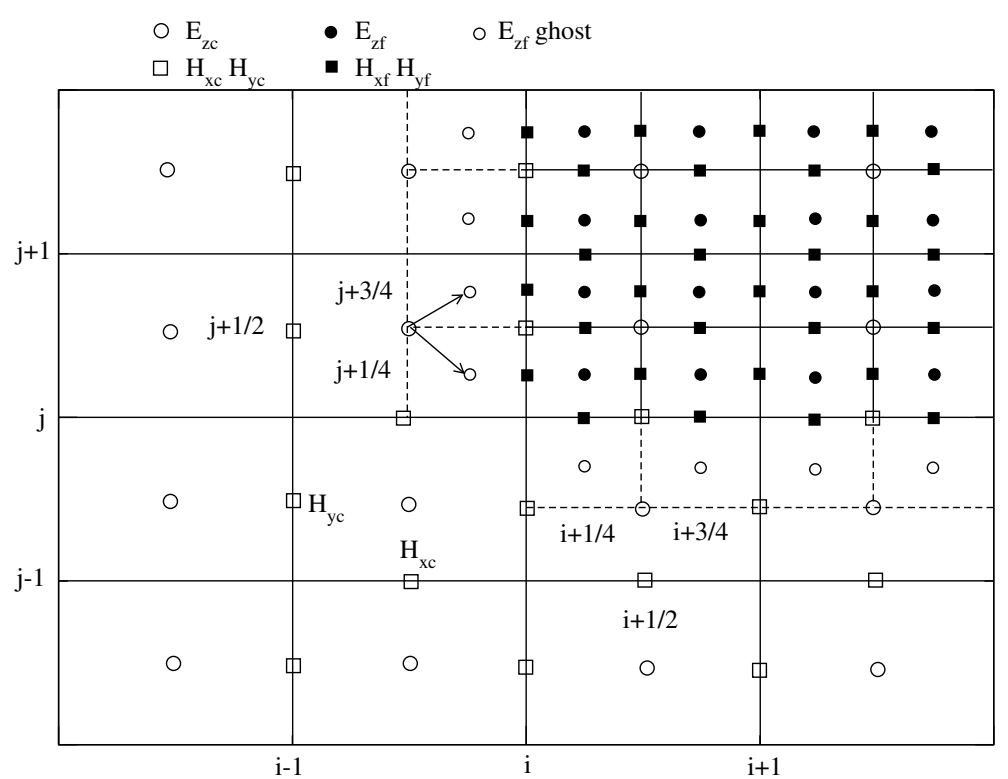

FIG. 2.1. Staggered grid with a 1:2 refinement interface. Dashed lines denote boundaries of the ghost cells. Arrows show a sample interpolation from coarse to fine $E_{z}$ electric field values.

In one space dimension, (dropping the $y$-dependence and $H_{x}$ component of the magnetic field), the numerical dispersion relation of the Yee scheme is

$$
\sin \left(\frac{\omega \Delta t}{2}\right)= \pm \nu \sin \left(\frac{k \Delta x}{2}\right),
$$

where $\Delta t$ and $\Delta x$ describe the grid size on the level of refinement considered.

When considering an initial value problem for Maxwell's equation, the wave number $k$ is given and the frequency $\omega(k)$ is determined by the dispersion relation. The 
stability condition, uniform boundness of the numerical solution with respect to $n-$ number of iterations and initial condition $e^{i k x}$, for $k \in\left[-\frac{\pi}{\Delta x}, \frac{\pi}{\Delta x}\right]$, implies that $\nu<1$. The scheme is dispersive and nondissipative: $\omega(k)$ is real and amplification factor is unity for real $k$, with the phase velocity $\omega / k$ and group velocity $\omega^{\prime}(k)$ approximating exact phase and group velocities to second order accuracy near $k=0$. Note that the original wave equation describes a simple advection with the dispersion relation $\omega= \pm k$.

In the case of a boundary value problem or a problem with a forcing term that oscillates at a given frequency $\omega$, the wave number $k(\omega)$, determined by the above dispersion relation, becomes complex for values of $\omega$ larger than the cutoff frequency $\omega_{c}$, that is defined by identity $\sin \left[\omega_{c} \Delta t / 2\right]=\nu$. The phase and group velocities of the plane wave are shown in Fig. 2.2 computed for $\nu=0.4$ and $\Delta x=0.02 \mu m$. On the same figure it is shown that the wave amplitude attenuates exponentially for frequencies between the cutoff frequency and the Nyquist frequency, while the group velocity is zero in this regime that is often referred to. as a region of non-propagating modes.

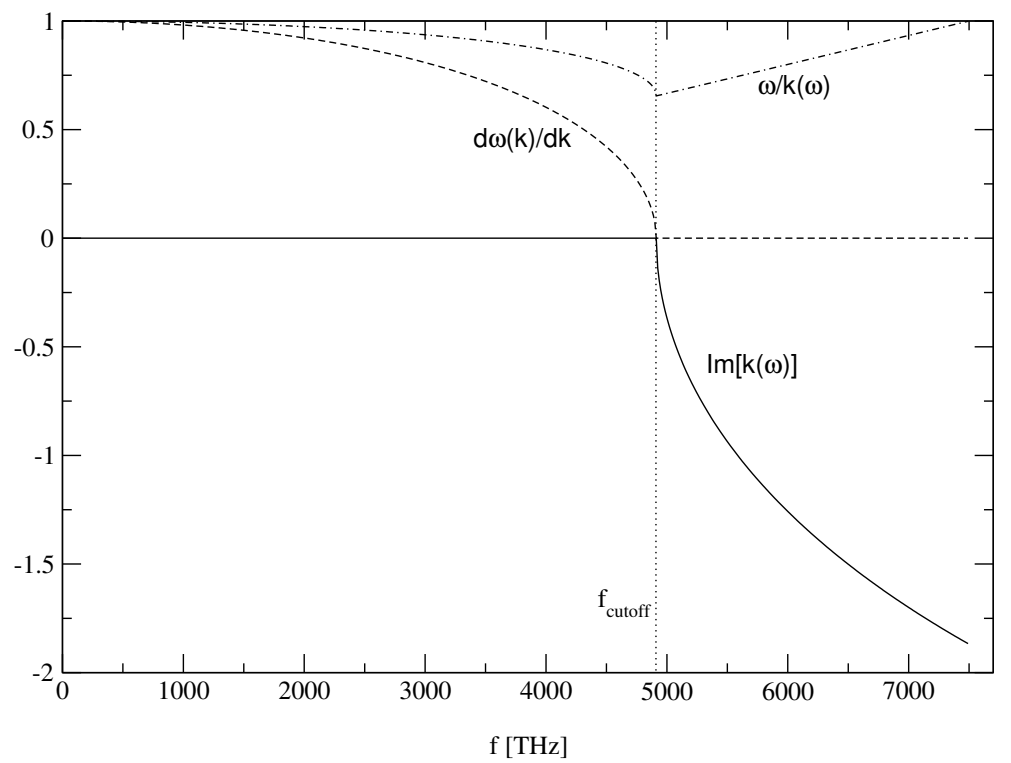

FIG. 2.2. Attenuation, phase and group velocities of the Yee scheme from the plane wave analysis.

On the fine grid the cutoff frequency is double that on the coarse grid if the grid size ratio is $1: 2$, as follows from eq. (2.4). Therefore on the fine grid, the relevant frequencies (up to the cutoff frequency) include both propagating and non-propagating modes of the coarse grid. As shown in the next section numerical difficulties arise beyond the cutoff frequency of the coarse grid due to the reflection of these nonpropagating modes back into the fine grid.

2.2. AMR Algorithm. The grid refinement algorithm is designed in the finite volume context with electric field representing the cell averages and magnetic field and its circulation defined along the cell boundaries, analogous to the flux function in the systems of hyperbolic conservation laws. The discussion is given for the TM mode, 
but it applies equally to the TE mode, and can be derived by exchanging the roles of the magnetic and electric fields.

Referring to the Fig. 2.1 and defining update as application of the Yee scheme (2.1)-(2.3), the algorithm can be summarized by the following steps:

0 . Update on the coarse grid, $n \rightarrow n+1$ :

$$
\begin{aligned}
& H_{x c i+1 / 2, j}^{n+1 / 2} \leftarrow H_{x c i+1 / 2, j}^{n-1 / 2}, \quad E_{z c i+1 / 2, j \pm 1 / 2}^{n} \\
& H_{y c i, j+1 / 2}^{n+1 / 2} \leftarrow H_{y c i, j+1 / 2}^{n-1 / 2}, \quad E_{z c i \pm 1 / 2, j+1 / 2}^{n} \\
& E_{z c i+1 / 2, j+1 / 2}^{n+1} \leftarrow E_{z c i+1 / 2, j+1 / 2}^{n}, \quad H_{y c i+1, j+1 / 2}^{n+1 / 2}, \quad H_{x c i+1 / 2, j+1}^{n+1 / 2} \cdots
\end{aligned}
$$

1. Interpolate ghost cell values $E_{z f i \pm 1 / 4, j \mp 1 / 4}^{n}$, etc. from $E_{z c}^{n}$ and $E_{z f}^{n}$

2. Update on the fine grid, $n \rightarrow n+1 / 2$ :

$$
\begin{aligned}
& H_{x f i+1 / 4, j}^{n+1 / 4} \leftarrow H_{x f i+1 / 4, j}^{n-1 / 4}, \quad E_{z f i+1 / 4, j \pm 1 / 4}^{n} \\
& H_{y f i, j+1 / 4}^{n+1 / 4} \leftarrow H_{y f i, j+1 / 4}^{n-1 / 4}, \quad E_{z f i \pm 1 / 4, j+1 / 4}^{n} \\
& E_{z f i+1 / 4, j+1 / 4}^{n+1 / 2} \leftarrow E_{z f i+1 / 4, j+1 / 4}^{n}, \quad H_{y f i+1 / 2, j+1 / 4}^{n+1 / 4}, H_{x f i+1 / 4, j+1 / 2}^{n+1 / 4}
\end{aligned}
$$

3. Interpolate ghost cell values $E_{z f i \pm 1 / 4, j \mp 1 / 4}^{n+1 / 2}$, etc. from $E_{z c}^{n}, E_{z c}^{n+1}$ and $E_{z f}^{n+1 / 2}$

4. Update on the fine grid, $n+1 / 2 \rightarrow n+1$ :

$$
\begin{array}{llll}
H_{x f i+1 / 4, j}^{n+3 / 4} \leftarrow & H_{x f i+1 / 4, j}^{n+1 / 4}, & E_{z f i+1 / 4, j \pm 1 / 4}^{n+1 / 2} \\
H_{y f i, j+1 / 4}^{n+3 / 4} & \leftarrow & H_{y f i, j+1 / 4}^{n+1 / 4}, & E_{z f i \pm 1 / 4, j+1 / 4}^{n+1 / 2} \\
E_{z f i+1 / 4, j+1 / 4}^{n+1} & \leftarrow & E_{z f i+1 / 4, j+1 / 4}^{n+1 / 2}, \quad H_{y f i+1 / 2, j+1 / 4}^{n+3 / 4}, & H_{x f i+1 / 4, j+1 / 2}^{n+3 / 4} \cdots
\end{array}
$$

5. Replace magnetic field on the coarse grid:

$$
\begin{aligned}
& H_{x c i+1 / 2, j}^{n+1 / 2}=\frac{1}{4}\left(H_{x f i+1 / 4, j}^{n+1 / 4}+H_{x f i+1 / 4, j}^{n+3 / 4}+H_{x f i+3 / 4, j}^{n+1 / 4}+H_{x f i+3 / 4, j}^{n+3 / 4}\right) \\
& H_{y c i, j+1 / 2}^{n+1 / 2}=\frac{1}{4}\left(H_{y f i, j+1 / 4}^{n+1 / 4}+H_{y f i, j+1 / 4}^{n+3 / 4}+H_{y f i, j+3 / 4}^{n+1 / 4}+H_{y f i, j+3 / 4}^{n+3 / 4}\right)
\end{aligned}
$$

6. Update interface cells $E_{z c i-1 / 2, j+1 / 2}^{n}$ and $E_{z c i+1 / 2, j-1 / 2}^{n}$, etc. as in step 0.

7. Replace $E_{z c}$ by the volume average of $E_{z f}$ in the regions where fine grid overlaps coarse grid.

These steps are applied recursively at each refinement level. Note that this approach is different from the one proposed in [3], where the grid refinement ratio of three is used and the magnetic field values at the interface are interpolated directly. In our formulation the electric field is interpolated to obtain the ghost cell values. Therefore, magnetic field values at the interface can be computed using the same Yee algorithm as in the interior. In contrast, the discrete energy conserving approach reported in [4] requires that fine and coarse values of the magnetic field be different on each side of the interface.

Further, the replacement of the magnetic field on the coarse grid by the spacetime averaged magnetic fields on the fine grid at the interface in step 5, enforces the preservation of the circulation across the grid hierarchy at multiples of the coarse time step, which can be seen from the following. For dielectric medium the Ampere's law, 
and its discrete counterpart, can be written in integral form as:

$$
\begin{aligned}
& \frac{\partial}{\partial t} \int_{A} \mathbf{D} d \mathbf{A}=\int_{l} \mathbf{H} d \mathbf{l} \\
& \sum_{i j} \epsilon_{i j}\left(\mathbf{E}_{i j}^{n+1}-\mathbf{E}_{i j}^{n}\right) \Delta x \Delta y / \Delta t=\sum_{l} \mathbf{H}_{l}^{n+1 / 2} d \mathbf{l} .
\end{aligned}
$$

Substituting the discrete time derivative from equation (2.3) into the left hand side of equation (2.6), and performing summation over all cells constituting the area $A$, shows that the discrete integral form of the Ampere's law is satisfied by the Yee scheme: all $\mathbf{H}$ fields cancel out except those that contribute to the integral of $\mathbf{H}$ along the boundary $l$ of $A$. We show that this property of the scheme holds in the presence of grid refinement, by considering an area $A$ that includes a refined grid region. The only modification to the sum occurs at the grid interfaces. With reference to Figure 2.1 the $\mathbf{E}$ field on the coarse grid is updated once from time $n$ to $n+1$ and $\mathbf{E}$ field on the fine grid is updated twice according to:

$$
\begin{aligned}
E_{z c i-1 / 2, j+1 / 2}^{n+1}-E_{z c i-1 / 2, j+1 / 2}^{n} & =\frac{\Delta t}{\Delta x}\left(H_{y c i, j+1 / 2}^{n+1 / 2}-H_{y c i-1, j+1 / 2}^{n+1 / 2}\right) \\
& -\frac{\Delta t}{\Delta y}\left(H_{x c i-1 / 2, j+1}^{n+1 / 2}-H_{x c i-1 / 2, j}^{n+1 / 2}\right), \\
E_{z f i+1 / 4, j+1 / 4}^{n+1 / 2}-E_{z f i+1 / 4, j+1 / 4}^{n} & =\frac{\Delta t}{\Delta x}\left(H_{y f i+1 / 2, j+1 / 4}^{n+1 / 4}-H_{y f i, j+1 / 4}^{n+1 / 4}\right) \\
& -\frac{\Delta t}{\Delta y}\left(H_{x f i+1 / 4, j+1 / 2}^{n+1 / 4}-H_{x f i+1 / 4, j}^{n+1 / 4}\right), \\
E_{z f i+1 / 4, j+1 / 4}^{n+1}-E_{z f i+1 / 4, j+1 / 4}^{n+1 / 2} & =\frac{\Delta t}{\Delta x}\left(H_{y f i+1 / 2, j+1 / 4}^{n+3 / 4}-H_{y f i, j+1 / 4}^{n+3 / 4}\right) \\
& -\frac{\Delta t}{\Delta y}\left(H_{x f i+1 / 4, j+1 / 2}^{n+3 / 4}-H_{x f i+1 / 4, j}^{n+3 / 4}\right),
\end{aligned}
$$

and similarly for $E_{z f i+1 / 4, j+3 / 4}^{n+1 / 2}$. By substituting equations (2.7)-(2.9) into the discrete integral in (2.6), and using for the coarse grid $\mathbf{H}$-fields on the interface (e.g. $\left.H_{y c i, j+1 / 2}^{n+1 / 2}\right)$ their values computed from space-time average in step 5 of the algorithm, again all $\mathbf{H}$-field values, except those on the boundary $l$, cancel out.

In step 1 (and 3 ) of the algorithm, ghost cell values of $E_{z f}$ are interpolated in space (and space-time) from nearby coarse and fine cells. One approach is to use second-order accurate linear interpolation with gradients $\nabla=\left(\nabla_{x}, \nabla_{y}\right)$ approximated by central differences, e.g. at the point $(i-1 / 2, j+1 / 2)$ in Fig. 2.1:

$$
\begin{aligned}
& \nabla_{x} E_{c z i-1 / 2, j+1 / 2}^{n}=\left(E_{c z i+1 / 2, j+1 / 2}^{n}-E_{c z i-3 / 2, j+1 / 2}^{n}\right) /\left(2 \Delta x_{c}\right), \\
& \nabla_{y} E_{c z i-1 / 2, j+1 / 2}^{n}=\left(E_{c z i-1 / 2, j+3 / 2}^{n}-E_{c z i-1 / 2, j-1 / 2}^{n}\right) /\left(2 \Delta y_{c}\right), \\
& E_{f z i-1 / 4, j+1 / 4}^{n}=E_{c z i-1 / 2, j+1 / 2}^{n}+\mathbf{d r}_{-} \cdot \nabla E_{c z i-1 / 2, j+1 / 2}^{n}, \\
& E_{f z i-1 / 4, j+3 / 4}^{n}=E_{c z i-1 / 2, j+1 / 2}^{n}+\mathbf{d r}_{+} \cdot \nabla E_{c z i-1 / 2, j+1 / 2}^{n},
\end{aligned}
$$

where $\mathbf{d} \mathbf{r}_{\mp}=\left(\Delta x_{c} / 4, \mp \Delta y_{c} / 4\right)$. The values of the ghost cells at time $n+1 / 2$ are obtained by additional linear interpolation in time:

$$
\begin{aligned}
& \nabla_{t} E_{c z i-1 / 2, j+1 / 2}^{n+1 / 2}=\left(E_{c z i-1 / 2, j+1 / 2}^{n+1}-E_{c z i-1 / 2, j+1 / 2}^{n}\right) / \Delta t_{c}, \\
& E_{f z i-1 / 4, j+1 / 4}^{n+1 / 2}=E_{f z i-1 / 4, j+1 / 4}^{n}+\left(\Delta t_{c} / 2\right) \nabla_{t} E_{c z i-1 / 2, j+1 / 2}^{n+1 / 2}, \\
& E_{f z i-1 / 4, j+3 / 4}^{n+1 / 2}=E_{f z i-1 / 4, j+3 / 4}^{n}+\left(\Delta t_{c} / 2\right) \nabla_{t} E_{c z i-1 / 2, j+1 / 2}^{n+1 / 2} .
\end{aligned}
$$


While second-order accurate, this interpolation method, as shown in section 2.3, results in a reflection coefficient $R$ larger than 1 for waves propagating from fine to coarse grid. In the following discussion we refer to this interpolation approach as linear.

A higher-order interpolation in space that involves a minimum number of points on the coarse grid can be obtained by using quadratic interpolation along each coordinate direction. As an example, at the point $(i-1 / 2, j+1 / 2)$ on Fig. 2.1, first a parabola in $y$ is used to obtain intermediate values at points $(i-1 / 2, j+1 / 4)$ and $(i-1 / 2, j+3 / 4)$, e.g.:

$$
E_{f z i-1 / 2, j+1 / 4}^{n}=\frac{5}{32} E_{c z i-1 / 2, j-1 / 2}^{n}+\frac{15}{16} E_{c z i-1 / 2, j+1 / 2}^{n}-\frac{3}{32} E_{c z i-1 / 2, j+3 / 2}^{n}
$$

Second, a parabola in $x$ interpolates to the position of the ghost cells, e.g.:

$$
E_{f z i-1 / 4, j+1 / 4}^{n}=\frac{8}{15} E_{f z i-1 / 2, j+1 / 4}^{n}+\frac{2}{3} E_{f z i+1 / 4, j+1 / 4}^{n}-\frac{1}{5} E_{f z i+3 / 4, j+1 / 4}^{n}
$$

In the following discussion we refer to this interpolation approach as quadratic. Alternatively, interpolation normal to the interface (along the $x$-axis) can be done using a linear approximation that involves one value of E-field from the fine grid:

$$
E_{f z i-1 / 4, j+1 / 4}^{n}=\frac{2}{3} E_{f z i-1 / 2, j+1 / 4}^{n}+\frac{1}{3} E_{f z i+1 / 4, j+1 / 4}^{n} .
$$

In the following discussion we refer to this interpolation approach as quadratic-linear.

Interpolation in time is done using linear interpolation, as in steps (2.10)-(2.11). Although the algorithm is given for a grid refinement ratio 1:2, it can be adapted for higher ratios. This, however, increases gradients in the grid size, which may affect stability. Then, several nested refinement levels may be preferable to one with high refinement ratio.

2.3. Reflection and transmission coefficients. The reflection and transmission coefficients were computed both analytically, using symbolic manipulations, and numerically - using the AMR computer program. The analytical solutions were obtained by substituting a plane wave ansatz into the interface boundary conditions and solving the resulting linear system for the unknown reflection and transmission coefficients.

2.3.1. One space dimension. The plane wave ansatz takes into account that a single coarse grid frequency $\omega \Delta t$ corresponds to two fine grid wavenumbers corresponding to the primary frequency $\omega \Delta t$ and the secondary aliased frequency $\tilde{\omega} \Delta t=\omega \Delta t+2 \pi$. The corresponding wavenumbers are denoted as $k(\omega \Delta t)$ and $\tilde{k}(\tilde{\omega} \Delta t)$. Note, that the secondary aliased wave is evanescent for all grid frequencies $\omega \Delta t \in[0, \pi]$, while the primary wave is propagating for grid frequencies $\omega \Delta t \in\left[0,4 \sin ^{-1}(\nu)\right]$. The exact solution to the reflection-transmission problem is obtained with the following plane wave ansatz,

$$
\begin{aligned}
& \left(\begin{array}{l}
E_{f z} \\
H_{f y}
\end{array}\right)=\left(\begin{array}{l}
1 \\
1
\end{array}\right) e^{i(k x+\omega t)}+R\left(\begin{array}{r}
1 \\
-1
\end{array}\right) e^{i(-k x+\omega t)}+\tilde{R}\left(\begin{array}{r}
1 \\
-1
\end{array}\right) e^{i(-\tilde{k} x+\tilde{\omega} t)}, \\
& \left(\begin{array}{c}
E_{c z} \\
H_{c y}
\end{array}\right)=T\left(\begin{array}{l}
1 \\
1
\end{array}\right) e^{i(k x+\omega t)}
\end{aligned}
$$


for a wave incident on the grid interface from the fine grid side $x>0$, and

$$
\begin{aligned}
& \left(\begin{array}{c}
E_{f z} \\
H_{f y}
\end{array}\right)=T\left(\begin{array}{r}
1 \\
-1
\end{array}\right) e^{i(-k x+\omega t)}+\tilde{T}\left(\begin{array}{r}
1 \\
-1
\end{array}\right) e^{i(-\tilde{k} x+\tilde{\omega} t)}, \\
& \left(\begin{array}{c}
E_{c z} \\
H_{c y}
\end{array}\right)=\left(\begin{array}{r}
1 \\
-1
\end{array}\right) e^{i(-k x+\omega t)}+R\left(\begin{array}{l}
1 \\
1
\end{array}\right) e^{i(k x+\omega t),}
\end{aligned}
$$

for a wave incident on the grid interface from the coarse grid side $x<0$. The reflection and transmission coefficients are determined by solving a linear system of equations obtained by substitution of the solution ansatz into the update equations for the ghost $\mathrm{E}$-field values and $\mathrm{H}$-fields at the interface.
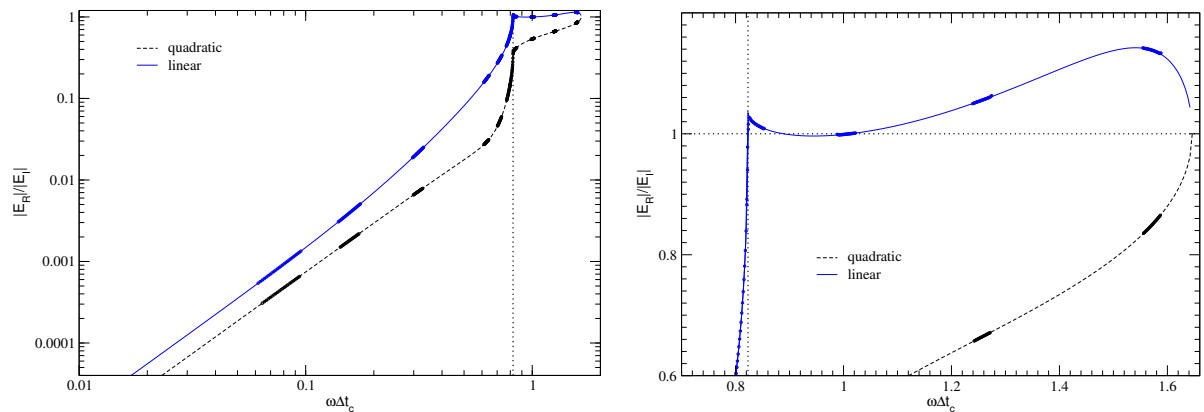

FIG. 2.3. Left: reflection coefficient in direction of fine to coarse. Right: enlarged region near cutoff frequency. Solid lines correspond to $R$ computed analytically using linear interpolation normal to the grid interface, dashed lines - quadratic interpolation normal to the grid interface. Symbols represent numerical solution. Vertical dotted line marks the cutoff frequency on the coarse grid.

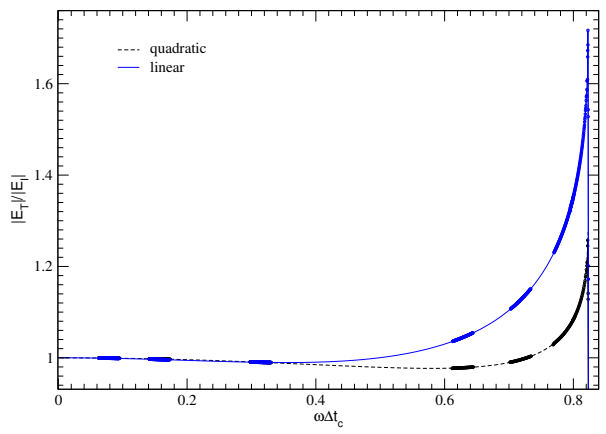

FIG. 2.4. Transmission coefficient in direction of fine to coarse.

Numerically measured reflection coefficients for the coarse (fine) reflected wave were computed by taking the difference between an incident Gaussian pulse $E_{z}=$ $\sin (2 \pi f t) \exp \left(-\left[\left(t-t_{0}\right) / \sigma\right]^{2}\right)$ with carrier frequency $f$, sampled near the grid interface on the (coarse) fine side, and a reference solution sampled at the same point, but computed without the interface. Then, the numerical reflection coefficient $R(f)$ was evaluated by taking a ratio of amplitudes of Discrete Fourier Transforms (DFT) of 
time samples of the two signals. The coarse to fine (fine to coarse) transmission coefficient $T(f)$ was evaluated by taking a ratio of DFTs of the transmitted signal on the fine (coarse) grid and a reference solution computed on the fine (coarse) grid without the interface.
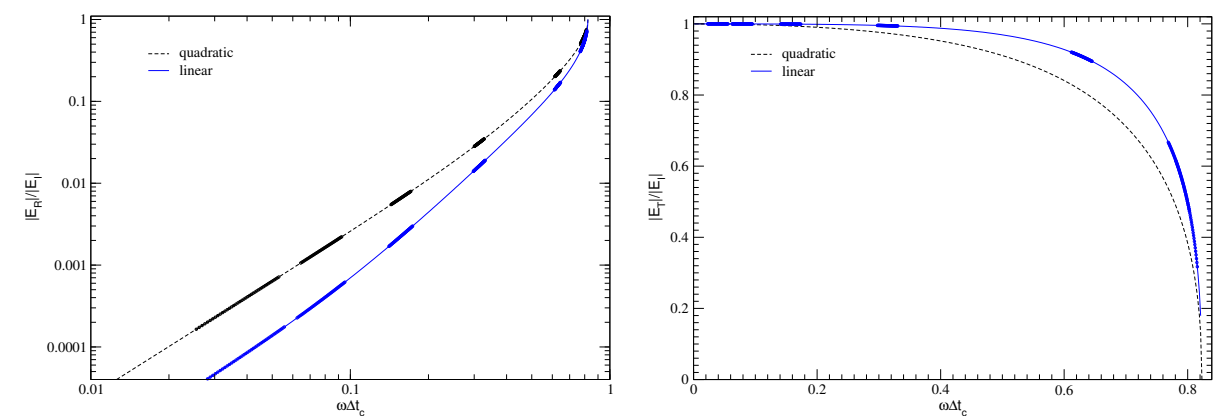

FIG. 2.5. Left: reflection coefficient in direction of coarse to fine. Right: transmission coefficient in direction of coarse to fine.

The reflection and transmission coefficients for fine-to-coarse propagation are shown in Figs. 2.3 and 2.4 for $\nu=0.4$. The numerically computed reflection coefficient is greater than 1 above the cutoff frequency of the coarse grid for the linear interpolation model, and less than 1 at all frequencies up to the Nyquist frequency of the coarse grid for the quadratic interpolation scheme. At low frequencies all methods recover second order accuracy, with the reflection coefficient $R \sim O\left(\Delta t^{2}\right)$. The transmission coefficient at the cutoff frequency is $T_{f c} \sim 1.3$ for the quadratic interpolation method, but the product of $T_{f c} T_{c f}$ is less than unity (Figure 2.5), and this prevents the build up of the energy in the high frequency modes.

2.3.2. Two space dimensions. In two dimensions on the fine grid there are two frequencies and two transverse wave numbers corresponding to single primary coarse frequency and transverse wave number: $\Delta t, \omega \Delta+2 \pi$ and $k_{y}, k_{y}+2 \pi$. This results in four possible reflected or transmitted waves on the fine side. The number of unknown coefficients corresponds to the number of interface conditions, for example, one coarse interface condition for the magnetic field corresponds to a single reflected/transmitted wave on the coarse grid, while two fine ghost values at each time sub-cycle of the fine mesh correspond to four reflected or transmitted waves on the fine grid. For a given frequency $\omega$ and transverse wavenumber $k_{y}$, the dispersion relation on both grids determines the wavenumber normal to the interface for the coarse and fine grids, respectively, with a corresponding dispersion relation on the coarse mesh,

$$
\begin{gathered}
\sin ^{2}\left(\frac{\omega \Delta t}{2}\right)=\nu^{2}\left[\sin ^{2}\left(\frac{k_{x} \Delta x}{2}\right)+\sin ^{2}\left(\frac{k_{y} \Delta y}{2}\right)\right] \\
\sin ^{2}\left(\frac{\omega \Delta t+2 \pi p}{4}\right)=\nu^{2}\left[\sin ^{2}\left(\frac{k_{x}^{p, q} \Delta x}{4}\right)+\sin ^{2}\left(\frac{k_{y} \Delta y+2 \pi q}{4}\right)\right] p, q=0,1,
\end{gathered}
$$


where we assumed that $\Delta x=\Delta y$ and therefore $\nu=\nu_{x}=\nu_{y}$. The amplitudes of the plane wave modes are normalized using the following variables,

$$
\begin{gathered}
A=\nu \frac{\sin \left(k_{x} \Delta x / 2\right)}{\sin ((\omega \Delta t / 2)}, B=-\nu \frac{\sin \left(\left(k_{y} \Delta y\right) / 4\right)}{\sin ((\omega \Delta t) / 4)}, \\
a_{p, q}=\nu \frac{\sin \left(k_{x}^{p, q} \Delta x / 4\right)}{\sin ((\omega \Delta t+2 \pi p) / 4)}, \quad b_{p, q}=\nu \frac{\sin \left(\left(k_{y} \Delta y+2 \pi q\right) / 4\right)}{\sin ((\omega \Delta t+2 \pi p) / 4)} .
\end{gathered}
$$

Then, the plane wave ansatz is

$$
\left(\begin{array}{c}
E_{f z} \\
H_{f y} \\
H_{f x}
\end{array}\right)=\left(\begin{array}{c}
1 \\
a_{0,0} \\
-b_{0,0}
\end{array}\right) e^{i\left(k_{x}^{0,0} x+k_{y} y+\omega t\right)}+\sum_{p, q} R_{p, q}\left(\begin{array}{c}
1 \\
-a_{p, q} \\
-b_{p, q}
\end{array}\right) e^{i\left(-k_{x}^{p, q} x+k_{y} y+\omega t\right)}
$$

with $p=0,1$ and $q=0,1$. The transmitted plane wave on the coarse side is given by

$$
\left(\begin{array}{l}
E_{c z} \\
H_{c y} \\
H_{c x}
\end{array}\right)=T e^{i\left(k_{x} x+k_{y} y+\omega t\right)}\left(\begin{array}{r}
1 \\
A \\
-B
\end{array}\right)
$$
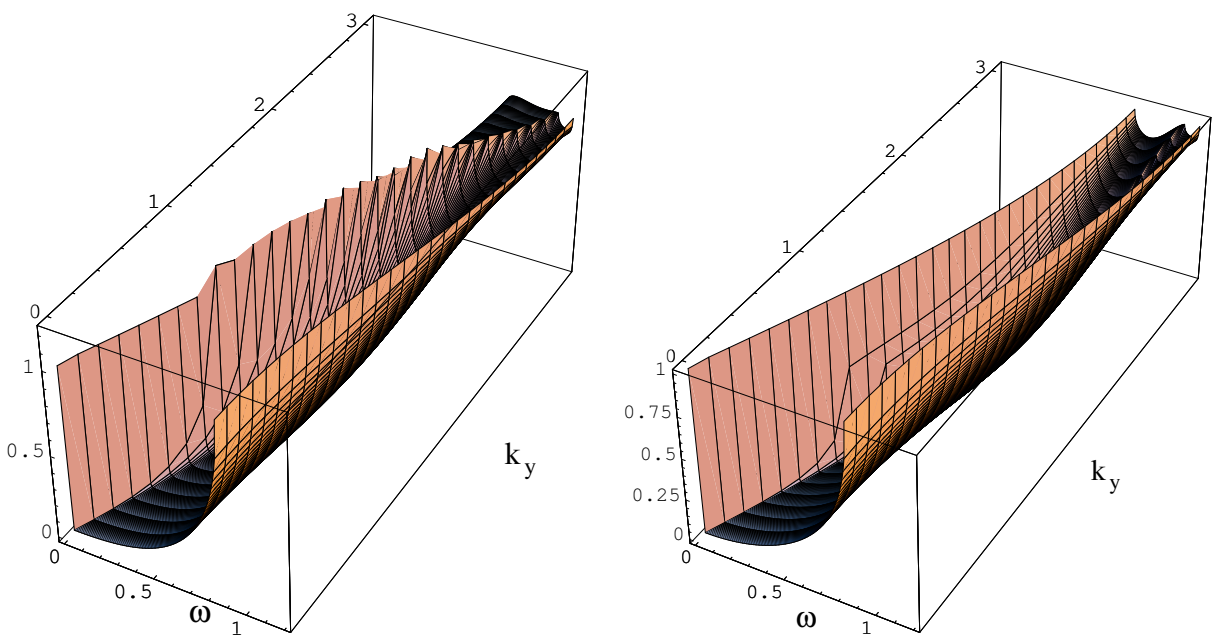

FIG. 2.6. Reflection coefficient $R\left(\omega, k_{y}\right)$ for waves incident from the coarse grid side in 2-D. Left: quadratic interpolation, right: linear interpolation.

Similarly, when the wave is incident onto an interface from the coarse grid side, $x<0$, there are four possible transmitted waves. The incident and reflected wave ansatz on the coarse side is,

$$
\left(\begin{array}{l}
E_{c z} \\
H_{c y} \\
H_{c x}
\end{array}\right)=\left(\begin{array}{r}
1 \\
-A \\
-B
\end{array}\right) e^{i\left(-k_{x} x+k_{y} y+\omega t\right)}+R\left(\begin{array}{r}
1 \\
A \\
-B
\end{array}\right) e^{i\left(k_{x} x+k_{y} y+\omega t\right)},
$$


On the fine side the transmitted wave ansatz is as follows,

$$
\left(\begin{array}{l}
E_{f z} \\
H_{f y} \\
H_{f x}
\end{array}\right)=\sum_{p, q} T_{p, q} e^{i\left(-k_{x}^{p, q} x+k_{y} y+\omega t\right)}\left(\begin{array}{c}
1 \\
-a_{p, q} \\
-b_{p, q}
\end{array}\right) e^{i\left(-k_{x}^{p, q} x+k_{y} y+\omega t\right)},
$$

where each of the variables $p$ and $q$ are equal to 0 or 1 .

In order to determine the five unknown reflection/transmission coefficients, the plane wave ansatz is substituted into the interface boundary conditions defined by interpolation equations of section 2.2. For example, using the linear interpolation equation (2.14), the planewave ansatz is substituted into following five equations, taking into account the staggered positioning of $\mathbf{E}$ and $\mathbf{H}$ fields in space and time:

$$
\begin{aligned}
& H_{x c i+1 / 2, j}^{n+1 / 2}=\frac{1}{4}\left(H_{x f i+1 / 4, j}^{n+1 / 4}+H_{x f i+1 / 4, j}^{n+3 / 4}+H_{x f i+3 / 4, j}^{n+1 / 4}+H_{x f i+3 / 4, j}^{n+3 / 4}\right) \\
& E_{z f i-1 / 4, j \mp 1 / 4}^{n}=\frac{2}{3} E_{z f i-1 / 2, j \mp 1 / 4}^{n}+\frac{1}{3} E_{z f i+1 / 4, j \mp 1 / 4}^{n} \\
& E_{z f i-1 / 4, j \mp 1 / 4}^{n+1 / 2}=E_{z f i-1 / 4, j \mp 1 / 4}^{n}+\left(E_{c z i-1 / 2, j+1 / 2}^{n+1}-E_{c z i-1 / 2, j+1 / 2}^{n}\right) / 2,
\end{aligned}
$$

where $E_{z f i-1 / 2, j \mp 1 / 4}^{n}$ is defined by interpolation along the grid interface in (2.13).

Plots of the absolute value of the reflection coefficient as a function of frequency and transverse wavenumber for quadratic and linear interpolation cases are shown in Figure 2.6. The transverse wavenumber $k_{y} \Delta y$ runs through interval $[0,2 \pi]$ taking into account that the normalization of the wavenumbers was done using the coarse grid $\Delta y$. The frequency interval is determined for each transverse wavenumber by the propagation condition of the incident wave, real $k_{x}^{(0,0)}$ or $0 \leq \sin \left(k_{x}^{(0,0)} \Delta x\right) \leq 1$ imposed by the respective dispersion relation.

Figure 2.6 shows that for the angles of incidence near $14^{\circ}$, the reflection coefficient for waves incident from the coarse grid side is larger than unity for the quadratic interpolation case. For the quadratic-linear scheme the reflection coefficient is $<1$ for all angles, preventing amplification of modes that undergo multiple reflections at the fine-coarse grid interface. A detailed analysis of the stability of the algorithm using other interpolation approaches for the two-dimensional methods, is presented in [2].

To estimate conservation properties of the algorithm, we integrated the solution using the grid structure shown in Figure 2.7 with the Gaussian inital condition and perfectly reflecting boundary conditions on the coarsest level. Divergence $\nabla \cdot \mathbf{B}$ computed on the coarse base grid is of the order of $10^{-6}$ for all times up to the $2 \times 10^{5}$ iterations that we tested.

The discrete approximation of electromagnetic energy $\epsilon\left(\mathbf{E}^{n}\right)^{2}+\left(\mathbf{H}^{n+1 / 2}\right)^{2} / \mu$ computed for the FDTD algorithm on a uniform grid (single level) and on a grid with 2 levels of refinement as in Figure 2.7 (left) is shown in Figure 2.8. The discrete energy on the uniform grid oscillates around its initial value, while on the refined grid it decays due to reduction in amplitude of the waves on each crossing of the grid interfaces. This smoothing is consistent with the second order accuracy of the scheme and accumulates to a noticeable amount after $10^{5}$ iterations. However this example contradicts the original aim of the grid refinement to capture solution gradients and energy on the finest grids, rather than allowing thousands of grid interface crossings. 

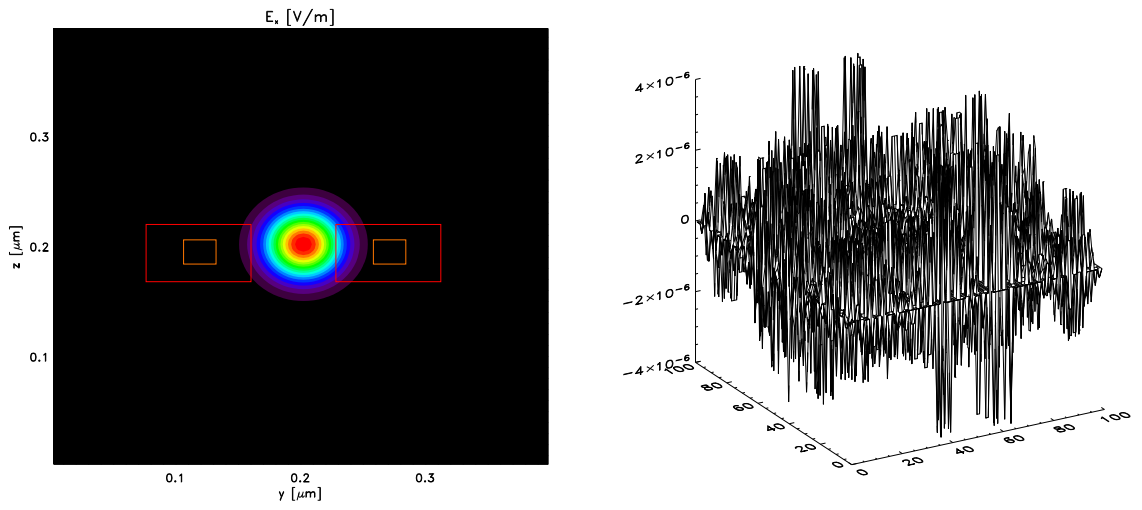

FIG. 2.7. Left: grid structure and initial condition used for the test of $\nabla \cdot \mathbf{B}=\mathbf{0}$. Right: $\nabla \cdot \mathbf{B}$ after $2 \times 10^{5}$ iterations on the coarse grid.

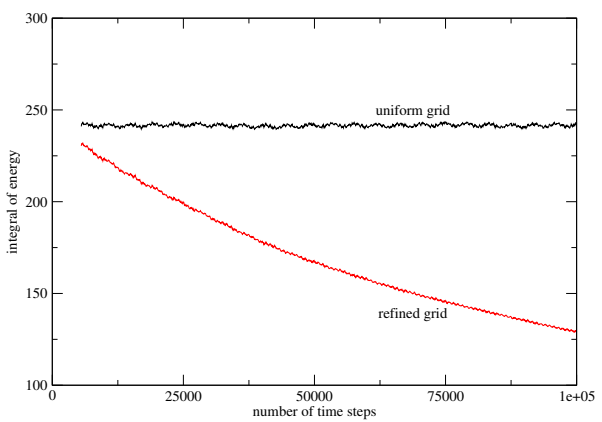

FIG. 2.8. Integral of discrete electromagnetic energy over the computational grid vs the number of time steps.

\section{Numerical Examples}

In the first example we consider a $2 \mathrm{D}$ optical waveguide with refractive index $n_{w}=3.2$ and width $w=0.3 \mu \mathrm{m}$. The waveguide is suspended in the background with index $n_{b}=1$, and extends along the $y$-axis from $y=0$ to $y=8.2 \mu m$, Figure 3.1.

One level of 1:2 grid refinement is used to surround the waveguide. The resolution on the coarse grid is $\Delta x=\Delta y=0.0272 \mu \mathrm{m}$. A carrier wave with frequency $200 \mathrm{THz}$ (free-space wavelength $\lambda=1.5 \mu \mathrm{m}$ ) is excited at $y=1.46 \mu \mathrm{m}$ with a transverse profile corresponding to the fundamental waveguide mode computed from the analytic dispersion relation. The ghost cell linear interpolation method becomes unstable. The left column of Fig. 3.1 shows that after ten thousand iterations the signal to noise ratio is about 0.3 . The high frequency waves that originate due to the discretization error, are trapped between the waveguide walls and the coarse-fine grid boundary. They experience multiple reflections and since the fine-to-coarse reflection coefficient in the linear interpolation case is greater than unity above the cutoff frequency, these modes are amplified, leading to instability. On the right side of Fig. 3.1 the field is shown for the quadratic interpolation method, with $R<1$ for all frequencies. The numerical solution is stable for long integration times: $5 \times 10^{4}$ fs corresponds to $2.75 \times 10^{6}$ iterations. 

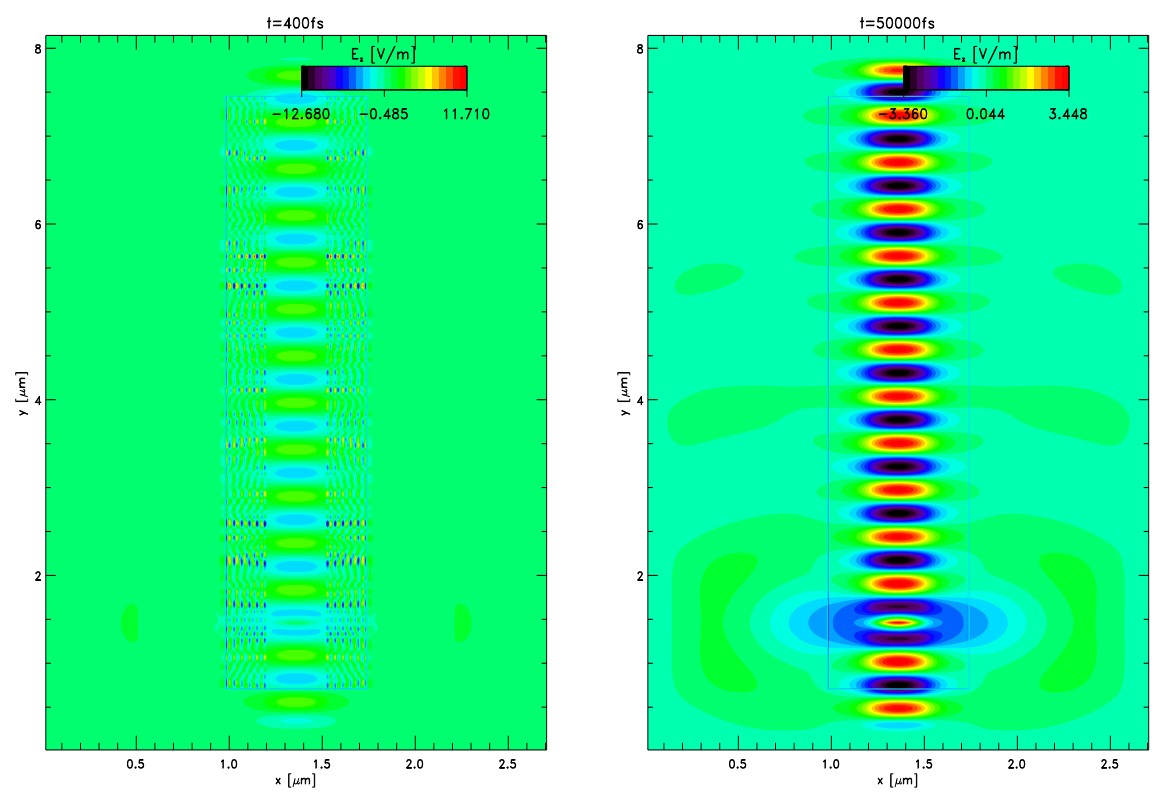

FIG. 3.1. Left: linear $O\left(\Delta x^{2}\right)$ interpolation, right: quadratic $O\left(\Delta x^{3}\right)$ interpolation.

The second example evaluates the rate of convergence of the method using quadratic interpolation. The solution is initialized with a Gaussian pulse,

$$
E_{z}(x, y)=\cos (2 \pi y) \exp \left[-\left(\left(x-x_{0}\right)^{2}+\left(y-y_{0}\right)^{2}\right) / \sigma^{2}\right]
$$

$H_{x}=-E_{z}, H_{y}=0, \sigma=0.8 \mu m, x_{0}=2.5 \mu m, y_{0}=3 \mu m$, that propagates along the $y$ axis in the background medium with refractive index $n_{b}=1$. A dielectric disk with index $n_{d}=2.0$ is set at $(x, y)=(2.5,6.0) \mu m$. The number of points on the coarsest grid is $\left(n_{x}, n_{y}\right)=(125,250)\left(\Delta x=\Delta y=0.04, N_{p p w}=25\right)$. Two levels $l=1,2$ of $1: 2$ grid refinement are used to resolve the disk boundary and a twenty point PML boundary layer terminates the coarse grid at level $l=0$. The wave diffracts in the $x$ direction as it propagates and interacts with the disk. Fig. 3.2 shows a snapshot of the electric field distribution at the time when the peak of the wave crosses the grid interface of the first refinement level. At the final time the incident wave has crossed the grid boundaries in both coarse-to-fine and fine-to-coarse directions.

Fig. 3.3 shows the differences between solutions at consecutive resolutions of $\Delta x$ and $\Delta x / 2$ in the discrete $L_{2}$ norm. We keep the staircased geometry invariant when changing the grid resolution, since our objective is to evaluate the errors due to the grid refinement interfaces and not staircased geometry. Solutions on both uniform and refined grids with one level of refinement are compared at $t=16$ fs on the domain $[1.0,4.0] \times[1.0,9.0] \mu m$, using values sampled on the coarse grid. In the case of the refinement the coarse values are replaced by the averages of the fine values according to the step 7 of the algorithm. As seen from the figure, in all cases the rate of convergence is second order, and the error of the solution obtained on the refined grid is approximately the same as error on the uniform grid with doubled resolution. The second-order errors due to grid interfaces can be seen from the log-scale plots of the reflection coefficients vs frequency, Figures 2.3,2.5. 

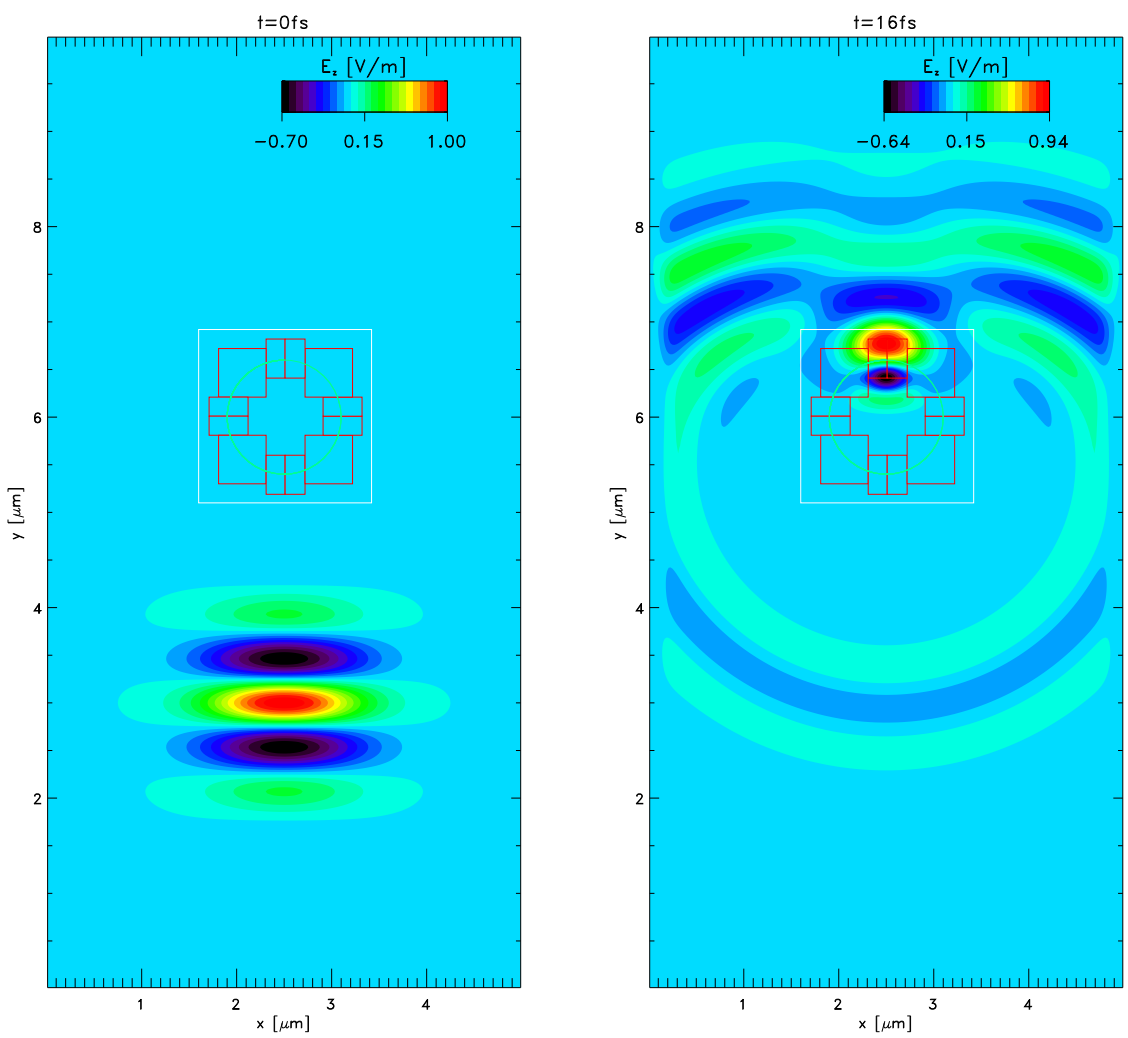

FIG. 3.2. Gaussian pulse incident on a dielectric disk. Boundaries of the dielectric and of the refined grids are outlined.

In the last example we compute the coupling efficiency and transmittance spectrum of the microcavity ring resonator from [9]. The geometry and the grid structure are shown in Fig. 3.4. The outer ring radius is $2.5 \mu \mathrm{m}$ and all waveguides have a width of $w=0.3 \mu \mathrm{m}$. The separation between straight waveguides and the ring section shown in Fig. 3.4 is $g=0.2312 \mu m$. Refractive index of the waveguides and of the background is set to $n_{w}=3.2$ and $n_{b}=1.0$ respectively. The cell size of the most refined grid is $\Delta x=\Delta y=0.0136 \mu \mathrm{m}$. A Gaussian pulse with carrier frequency 200 $\mathrm{THz}$ is initiated in the left waveguide $W_{L}$ using precomputed time dependence of the transverse electric field $E_{z}$ [9]. The pulse propagates and couples some of its energy into the ring microcavity, Fig. 3.5. This "trapped" pulse repeatedly circumnavigates the ring and couples its energy back into the waveguides $W_{L}$ and $W_{R}$ on each pass. To compute the coupling efficiency of the ring cavity, we use the same procedure as in [9]. First the Discrete Fourier Transforms of the time dependent $\mathbf{E}$ and $\mathbf{H}$ fields are calculated. The sampling of the fields is taken across the waveguide $W_{L}$ at $y=2.5 \mu \mathrm{m}$ and across the top section of the ring at $x=5.2 \mu \mathrm{m}$. Then, we integrate the Poynting vector across the waveguide cross-sections, and take the ratio of the resulting fluxes.

The computed coupling coefficient $\kappa$ for different gap sizes $g$, using uniform and refined grids with one and two levels of refinement, is shown in Fig. 3.6 as a function of frequency. The coupling coefficient computed using the multilevel grid structure 


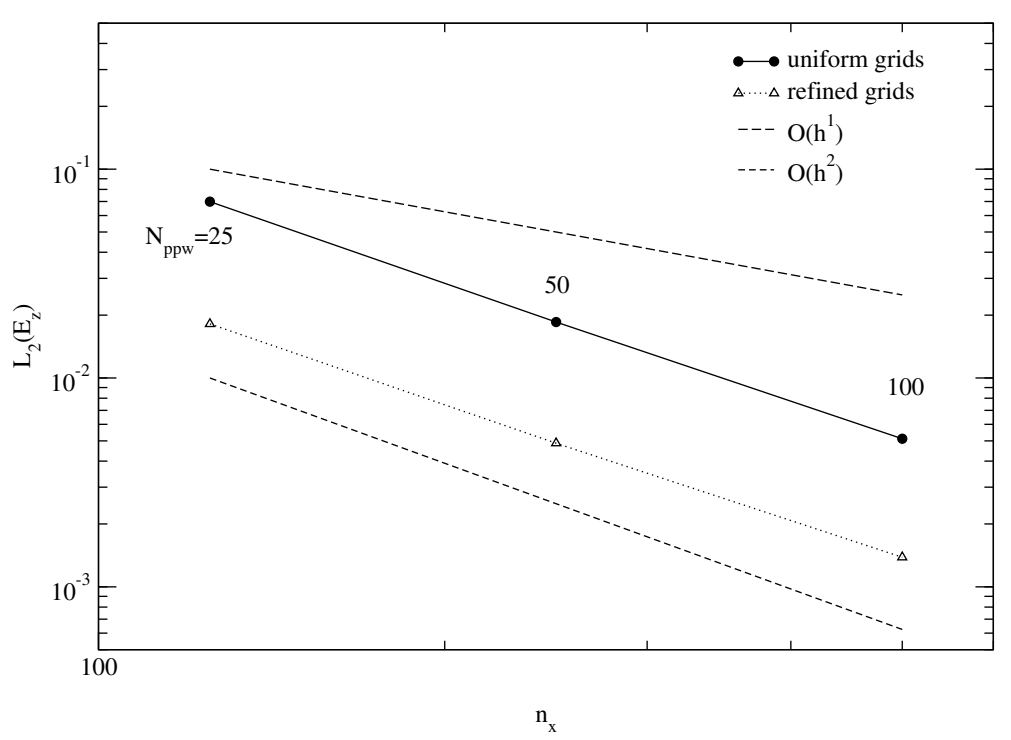

FIG. 3.3. $L_{2}$ error vs number of grid points. Long and short dashed lines correspond to ideal first- and second-order accurate scaling. $N_{p p w}$ indicates number of points per wavelength.

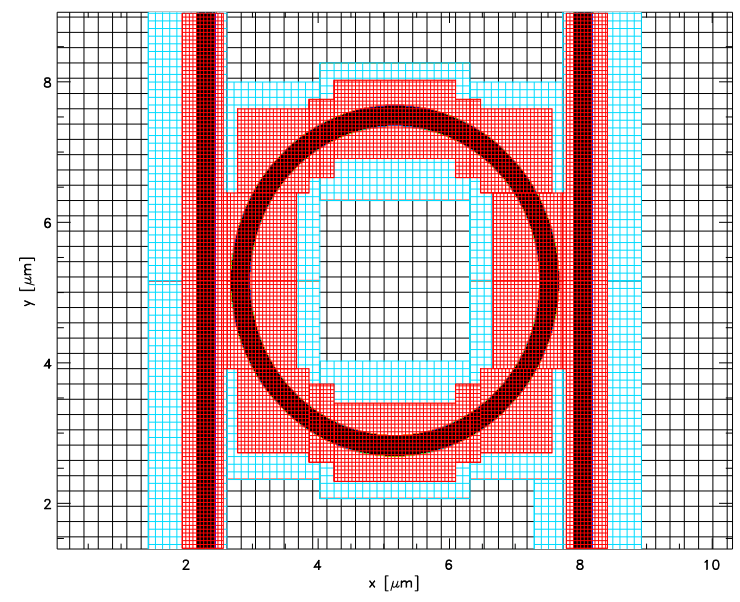

FIG. 3.4. Ring microcavity resonator geometry and grid structure. For clarity, grid lines are decimated by a factor of four.

agrees well with the values obtained on a uniform grid. The improvement in performance due to space and time mesh refinement depends on the number of refinement levels and the fraction of the computational domain that is refined. Table 3.1 compares required computer memory and normalized run times for the examples of pulse scattering from a dielectric rod and the microring cavity computations, to achieve relative accuracy of $10^{-3}$. Comparison is done for the case of uniform grids and refined 

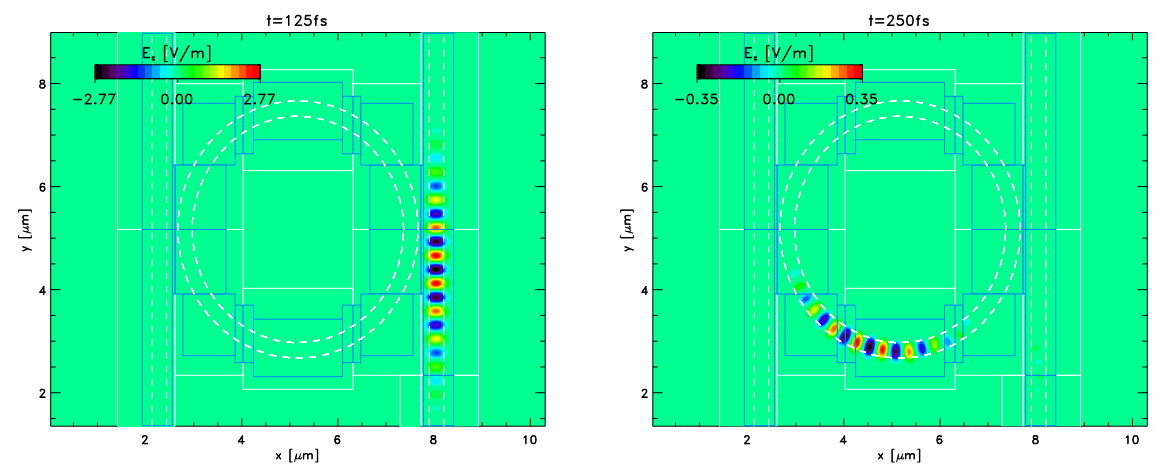

FIG. 3.5. Time evolution of the electric field $E_{z}$ component in the ring microcavity. Dashed lines mark waveguide boundaries.

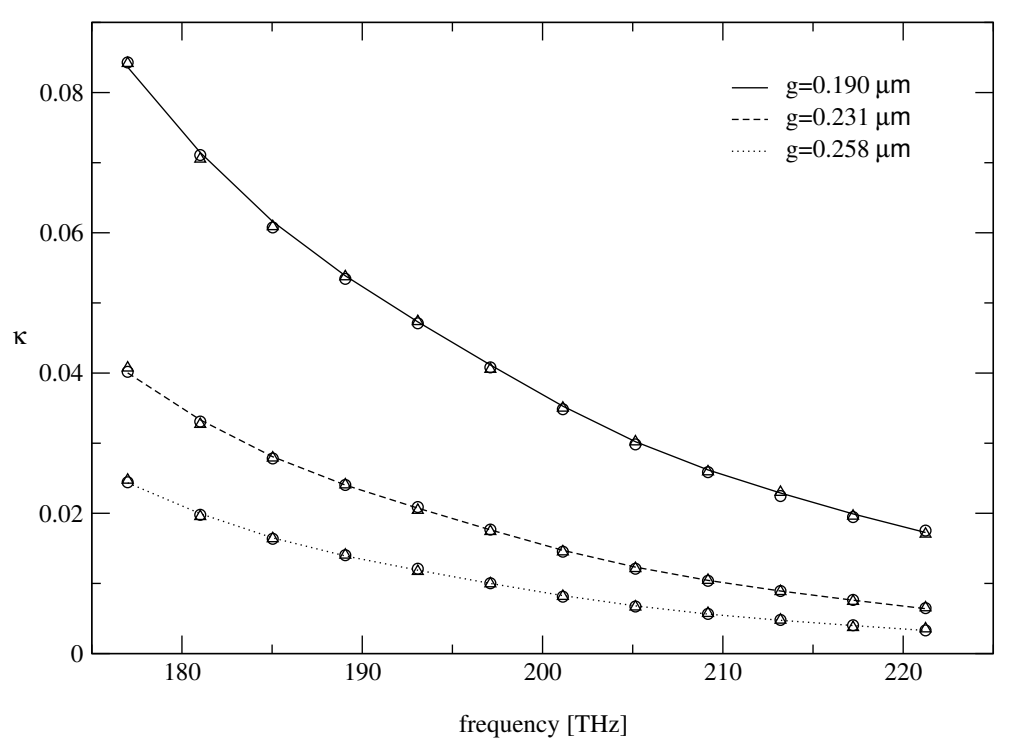

FIG. 3.6. Coupling efficiency of the microring as a function of frequency and gap size $g$. Lines correspond to uniform grid, circles - to one level, and triangles - to two levels of grid refinement.

grids with the same resolution on the most refined level. Using one and two levels of refinement for the microring resonator problem results in about 1.8 and 4.0 times improvement in computation time. 
TABLE 3.1. Comparison of the FDTD method on the uniform and refined grids

\begin{tabular}{|c|c|c|c|c|}
\hline & Number of Levels & $\Delta[\mathrm{nm}]$ & Memory [MB] & Run time \\
\hline \hline scattering & 1 & 10 & 58.7 & 17.5 \\
\hline scattering & 2 & $20-10$ & 17.8 & 3.4 \\
\hline scattering & 3 & $40-20-10$ & 6.3 & 1.0 \\
\hline microring & 1 & 13.6 & 78.4 & 3.8 \\
\hline microring & 2 & $27.2-13.6$ & 34.0 & 1.76 \\
\hline microring & 3 & $54.4-27.2-13.6$ & 28.6 & 1.0 \\
\hline
\end{tabular}

\section{Conclusions}

In this paper we have proposed a simple interface boundary condition for local space-time grid refinement appropriate for the FDTD method for Maxwell's equations in two space dimensions. The algorithm is in a form similar to the flux formulation of the hyperbolic conservation laws and allows for the use of previously developed data management algorithms for solution adaptive grid refinement. The algorithm preserves overall accuracy of the solution in the regions of high gradients, and is more efficient compared to the uniform grid case.

The interface boundary condition we propose is significantly simpler than the ones used previously. The numerical experiments demonstrate the accuracy, efficiency and stability of the algorithm for long time integration. Its applicability in three spatial dimensions and to dispersive material models is under investigation.

Acknowledgments. This work was supported in part by AFOSR contract F49620-03-1-0194.

\section{REFERENCES}

[1] M. J. Berger and R. J. LeVeque, Adaptive mesh refinement using wave-propagation algorithms for hyperbolic systems, SIAM J. Numer. Analysis, 35, 2298-2316, 1998.

[2] M. Brio, A. R. Zakharian, C. Dineen and J. V. Moloney, Stability of algorithms with local mesh refinement for Maxwell's equations, in preparation, 2004.

[3] M. W. Chevalier, R. J. Luebbers, FDTD local grid with material traverse, IEEE Tran. Anten. Prop., 45, 411-421, 1997.

[4] F. Collino, T. Fouquet, and P. Joly, A space-time mesh refinement method for the 1D Maxwell's equation, C. R. Acad. Sci. Paris, 328, 263-268, 1999.

[5] I. S. Kim, W. J. R. Hoefer, A local mesh refinement algorithm for the time-domain finite difference method to solve Maxwell's equations, IEEE Tran. Microwave Theory Tech., 38, 812-815, 1990.

[6] R. J. LeVeque, O. Steiner and A. Gautschy, Computational Methods for Astrophysical Fluid Flow, Berlin ; New York : Springer, 1998.

[7] P. Monk, Sub-gridding FDTD schemes, Applied Computational Electromagnetics Society J., 11, 37-46, 1996.

[8] M. Okoniewski, E. Okoniewska and M.A. Stuchly, Three dimensional subgridding algorithm for FDTD, IEEE Tran. Ant. and Prop., 45, 422-429, 1997.

[9] A. Taflove and S. C. Hagness, Computational Electrodynamics: the FDTD method, 2nd ed., Boston ; London : Arthech House, 2000.

[10] P. Thoma and T. Weiland, A consistent subgridding scheme for the finite difference time domain method, Inter. J. Numer. Modeling, 9, 359-374, 1996.

[11] J.-L. Vay, An extended FDTD scheme for the wave equation: application to multiscale electromagnetic simulation, J. Comp. Phys., 167, 72-98, 2001. 